\title{
Radix entomolaris: a case report
}

\author{
Viktoria Petrova, Radosveta Vassileva, Sevda Yantcheva \\ Department of Conservative Dentistry, Faculty of Dental \\ Medicine, Medical University, Sofia, Bulgaria
}

\begin{abstract}
Introduction: Knowing root canal anatomy with all its exceptions is a key factor for the success of the endodontic treatment. Radix entomolaris is an anatomical variation of lower first molars which is very rarely found in patients from the European race.

Case report: A clinical case of a 28-years old patient with radix entomolaris is presented. Dental operating microscope and standard sector radiographs were used for the diagnostics and treatment of tooth 36 . Root canals were instrumented with Ni-Ti rotary and reciprocating files. Clinical and radiographic healing was observed at the end of the first year after treatment.
\end{abstract}

Conclusion: Instrumentation of all existing root canals and their three dimensional sealing leads to predictable clinical result of the endodontic treatment. Good knowledge, usage of magnification and contemporary endodontic instruments aid the dentist in the treatment of complex clinical cases like radix entomolaris.

Keywords: anatomical variations, radix entomolaris, endodontic treatment

\section{Background}

The success of the endodontic treatment depends on finding of all existing root canals, their cleaning, shaping and three dimensional sealing (1). Knowing of root canal anatomy with all its variations is a key factor.

Lower first molars are the first to erupt in the permanent dentition. They are also the first to be affected by caries and its complications leading to the necessity of endodontic treatment. Usually lower first molars have two roots and three or four root canals (2). Very seldom these teeth may have a supernumerary root which can be situated mesially (radix paramolaris) or distally (radix entomolaris) (3).

Carabelli (4) was the first one to describe the anatomical variation of lower sixth teeth having a supernumerary root. In 1915 Bolk names this variation radix entomolaris (5). Additional roots are connected with certain ethnicity or geographical location. The reasons for its appearance are not clear but can be a sign of atavism or disturbance during ontogenesis $(1,6)$. The frequency of this variation is different for the different ethnicities: in the Asian population it's between 5.8 and $33.1 \%(1,7,8)$, in the Ararbic - 2.3-6\% (7), in the African - 0.7-3.1\% (7), European - 0.7-3.3\% (7). 


\section{Case description}

A 28 years old European patient was referred to the dental office with pain in the lower left quadrant. He didn't report of any concomitant diseases. The patient complained of having had dull pain during pressure or chewing in the region of the first molar for the last 5-6 moths. Intraorally a bridge was present. The mucosa around the tooth apexes was one of normal color. The patient felt pain during percussion and palpation in the apical region of tooth 36. After panoramic and sector radiographs (fig.1) a diffuse flame-like lesion around the apexes of teeth 35,36 and 37 was observed. Tooth 36 was found to have the anatomical variation radix entomolaris. A separated file was present in its mesio-buccal root canal.

After signing written consent the bridge was removed. Tooth 36 was isolated with rubber dam. A dental operating microscope (DOM) SOM62/1100 (Karl Kaps GmbH \& Co. KG) was used during the endodontic treatment. Access cavity was prepared with a diamond bur and orifices were relocated. With the help of the magnification of the DOM and the use of an endodontic explorer (DG-16, Sybron Endo) the additional root and root canal observed on the radiographs were clinically found. Precurved K-files \#10 (Dentsply Maillefer) were used for initial root canal negotiation.

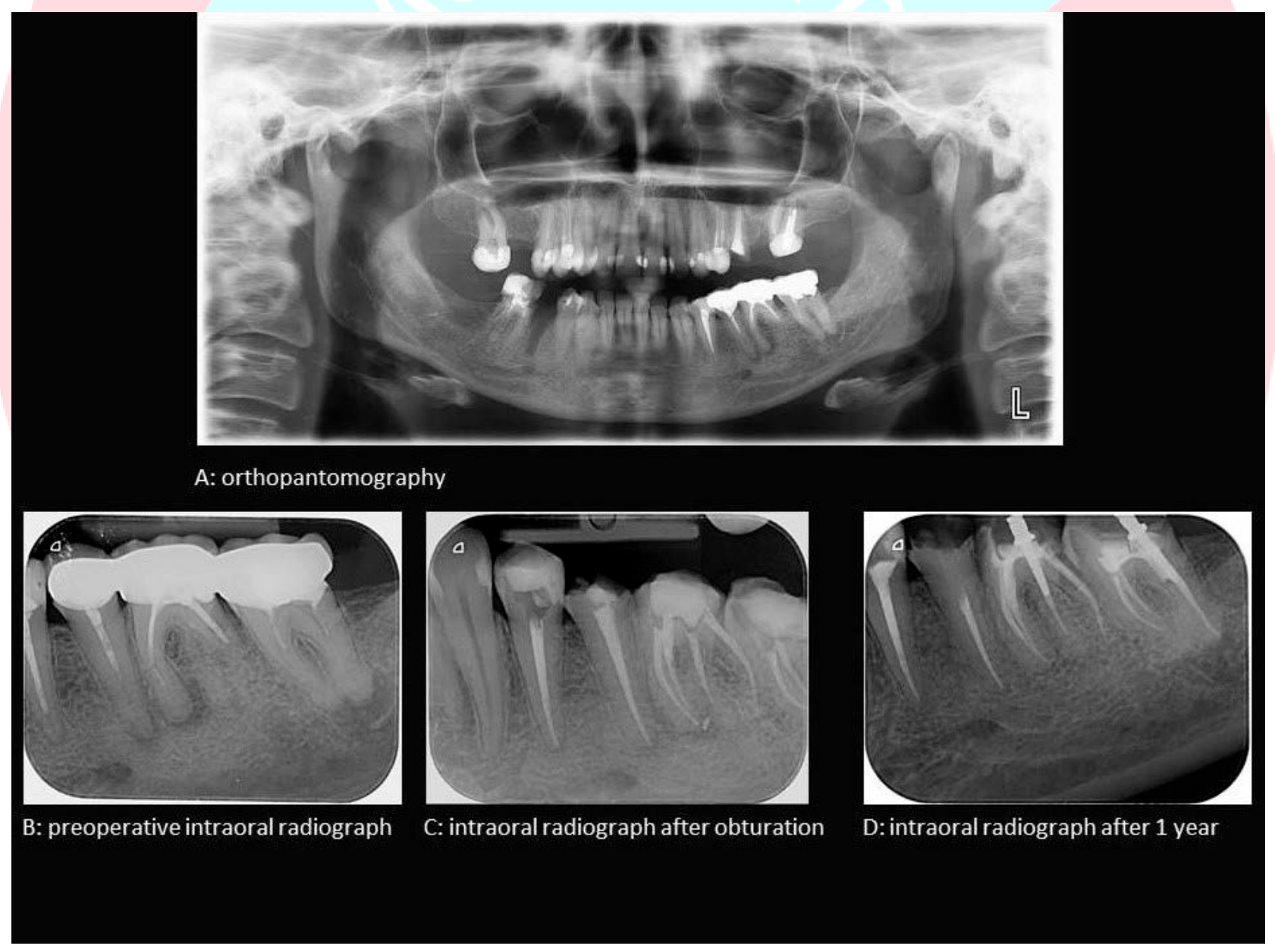

Fig.1. Radiograph of tooth 36: A. Ortopantomography B. Preoperative intraoral periapical radiograph C. Intraoral periapical radiograph after obturation D. Intraoral periapical radiograph after 1 year 
The previously discovered fractured instrument in the MB root canal was successfully bypassed with a C+ file \#10 (Dentsply Maillefer). For the glide path the S1 file from the ProTaper Universal System was used (Densply Maillefer). The MB, ML and DL root canals were instrumented using Wave One Gold Primary and Wave One Gold Medium (Dentsply-Maillefer) was used for the DB canal.

The irrigation protocol that was applied included $5.25 \% \mathrm{NaOCl}, 17 \%$ EDTA and physiological solution. For additional activation of the irrigating solutions ultrasonic files and Endo Activator (Dentsply Maillefer) were used in each root canal for 60 seconds each. After instrumentation $\mathrm{Ca}(\mathrm{OH}) 2$ was applied in the root canals. Permanent sealing of the root canals was performed after 10 days. $\mathrm{H}$-files and Ultrasonic files were used to remove $\mathrm{Ca}(\mathrm{OH}) 2$. The canals were irrigated with physiological solution and dried with paper points corresponding to the last used instrument on the full working length for the preparation of the canals. Root canals were sealed using eugenol-based sealer and gutta-percha cones with the single cone technique. After cleaning the endodontic cavity orifices of root canals were adhesively sealed with a flowable resin composite and the patient was referred back to his general practitioner dentist for the final restoration of the tooth. After a year a follow-up radiograph was made.

\section{Discussion}

Depending on the ethnicity radix entomolaris can be commonly or very rarely found. In the European race to which the patient belonged this anatomical variation is rare. We found very few similar cases in the literature (7).

Clinical and radiographic signs are used for the discovery of radix entomolaris. The presence of an additional cusp points to the presence of a supernumerary distal root. The shape of the pulp chamber and application of the principle of symmetry can help for the discovery of radix entomolaris (9). Other clinical diagnostic methods that can aid are periodontal probing - for the discovery of the additional root, and the "champagne effect" - appearance of bubbles in the pulp chamber showing another orifice after placing hypochlorite (10). The usage of magnification considerably enhances visibility and the ability of the dentist to discover different anatomical variations (11). In the presented clinical case a dental operating microscope was used, which is proved to help discover additional root canals.

Radiographs and CBCT are paraclinical methods which can help locate radix entomolaris $(1,12,13)$. Orthopantomographic and sector radiographs have image superposition which can sometimes make discovery of the additional root impossible. СBCT is a contemporary method which is widely used in endodontics and gives clear visibility not only for additional root canals but also for their curvatures. Although СВCT can give very useful information it has greater beam load than conventional radiographs. In the presented clinical case we used only conventional radiographic methods because radix entomolaris was clearly visible with them. According to studies in $90 \%$ of the cases described in the literature the intraoral periapical radiograph is completely sufficient to discover a supernumerary root $(1,6,9)$. In other in vivo studies CBCT was necessary to be used $(7,12)$.

According to De Moor et al (14) radix entomolaris can be divided into three types:

Type I - straight root and root canal

Type II - coronal curve followed by a straight root and root canal

Type III - coronal curve followed by an additional curve located between the middle and apical third CBCT can give the best information for the specific typization and root canal curvatures of radix entomolaris. 
The orifice of radix entomolaris root canal is usually located in mesio-lingual direction to the main distal canal. This imposes a change in the shape of the access cavity - from triangular to trapezoid to provide straight line access to the canal. In cases of severely curved canals initial precurving of the stainless steel files is recommended to be able to reach the apical third $(6,9)$. In the presented clinical case we used Nickeltitanium reciprocating instruments form the system Wave One Gold. These instruments are single used which considerably decreases the risk of separation. The new technology for treatment of the $\mathrm{Ni}-\mathrm{Ti}$ alloy called "gold" increases the flexibility and reduces the cyclic fatigue of the material (15). The reciprocating movement of these files is safer and preferred during instrumentation of curved root canals as are in most of the cases the canals of radix entomolaris (16).

\section{Conclusion}

Knowing of tooth and root canal anatomy with all its exceptions increases the success rates of root canal treatment. Radix entomolaris is an extremely rarely found variation of the lower first molar in patients from the European race. Preliminary radiographic diagnostics, the correct shape of the access cavity, the usage of magnification and the choice of instruments suitable for curved root canals, as radix entomolaris are in most of the cases, leads to successful endodontic treatment.

\section{Acknowledgment}

This article is sponsored by the Scientific Council of Medical University - Sofia, Bulgaria, Infrastructural Project No 5021/10.07.2019; Contract No D-236/19.12.2019.

\section{References}

1. Attam K, Nawal RR, Utneja S, et al. Radix entomolaris in mandibular first molars in Indian population: a review and case reports. Case Rep Dent. 2012;2012:595494. doi: 10.1155/2012/595494.

2. Vertucci FJ. Root canal anatomy of the human permanent teeth. Oral Surg Oral Med Oral Pathol. 1984;58:589-599.

3. Kuzekanani M, Walsh LJ, Haghani J, et al. Radix Entomolaris in the Mandibular Molar Teeth of an Iranian Population. Int J Dent. 2017;2017:9364963. doi: 10.1155/2017/9364963.

4. Carabelli G. Systematisches Handbuch der Zahnheilkunde. 2nd ed. Vienna: Braumuller und Seidel; 1844. p. 114.

5. Bolk L. Bemerkungen über wurzelvariationen am menschlichen unteren molaren. Zeitschrift für Morphol und Anthropol. 1915;3:605-610.

6. Walker RT, Quackenbush LE. Three-rooted lower first permanent molars in Hong Kong Chinese. Br Dent J 1985;9:298-299.

7. López-Rosales E, Castelo-Baz P, De Moor R, Ruíz-Piñón M, Martín-Biedma B, Varela-Patiño P. Unusual root morphology in second mandibular molar with a radix entomolaris, and comparison between cone-beam computed tomography and digital periapical radiography: a case report. J Med Case Rep. 2015 Sep 22;9:201. doi: 10.1186/s13256-015-0681-x.

8. Yew SC, Chan K. A retrospective study of endodontically treated mandibular first molars in a Chinese population. J Endod 1993; 19(9):471-473. 
9. Sinha D, Mahesh S, Jaiswal N. Radix entomolaris: A report of two cases. Bull Tokyo Dent Coll. 2016; 57(4):253-258.

10. Hegde V, Kashid V. Radix entomolaris-series of case reports, Int J Adv Case Rep 2015;2(4):216-220.

11. Perrin P, Neuhaus KW, Lussi A. The impact of loupes and microscopes on vision in endodontics. Int Endod J. 2014;47(5):425-429.

12. Aggarwal $V$, Singla $M$, Logani A, et al. Endodontic management of a maxillary first molar with two palatal canals with the aid of spiral computed tomography: a case report. J Endod 2009;35(1):137-139.

13. Duman SB, Duman S, Bayrakdar IS, et al. Evaluation of radix entomolaris in mandibular first and second molars using cone-beam computed tomography and review of the literature. Oral Radiol. 2020 Oct;36(4):320-326. doi: 10.1007/s11282-019-00406-0.

14. De Moor R, Deroose C, Calberson F. The radix entomolaris in mandibular first molars, an endodontic challenge. Int Endodo J 2004;37(11):789-799.

15. Canali L, Duque JA, Vivan RR, et al. Comparison of efficiency of the retreatment procedure between Wave One Gold and Wave One systems by Micro-CT and confocal microscopy: an in vitro study. Clin Oral Investig 2019 Jan;23(1):337-343. https://doi.org/10.1007/s00784-018-2441-y.

16. Shen Y, Cheung GS, Bian Z, et al. Comparison of defects in profile and ProTaper systems after clinical use. J Endod 2006;32:61-65.

\section{Corresponding author:}

Dr. Viktoria Petrova,

Department of Conservative Dentistry, Faculty of Dental Medicine, Medical University, Sofia;

01, St. Georgi Sofiiski blvd., 1431 Sofia, Bulgaria.

Tel: +359 895431291,

E-mail: viktoriapetrova@abv.bg 